PAEDIATRICS

\title{
Fruitful therapy for dehydration
}

Dilute apple juice is superior to electrolyte maintenance solution for the treatment of minimal dehydration in children with mild gastroenteritis, according to a new clinical trial published in JAMA.

Gastroenteritis is an extremely common occurrence in children, and frequently results in dehydration owing to vomiting and diarrhoea. Gold-standard treatment for these patients comprises oral or, in more severe cases, intravenous rehydration solutions.

"Many of these children's caregivers try to administer oral electrolyte maintenance solutions at home but the children either refuse to drink them or they continue to vomit," explains first author Stephen Freedman. "Often, children receive intravenous rehydration despite not being substantially dehydrated, and in our study it appeared that this was often a response to a failed oral rehydration challenge in the emergency department. A less dogmatic approach aimed at providing fluids that children like might overcome these problems."

To investigate their hypothesis, Freedman and colleagues conducted a randomized, single-blind noninferiority trial of dilute apple juice - chosen due to its popularity with children - versus apple-flavoured oral electrolyte maintenance solution in children (aged 6-60 months) with mild gastroenteritis and minimal dehydration. Treatment failure (defined by any of a composite of measures, including necessity of intravenous rehydration, hospitalization and protracted symptoms, occurring within 7 days of enrolment) was the primary outcome.

Children receiving apple juice $(n=323)$ experienced less treatment failure than those given electrolyte maintenance solution ( $n=324)$, demonstrating the superiority of the alternative rehydration approach. Notably, only $2.5 \%$ of children administered apple juice required intravenous rehydration, compared with $9 \%$ of children receiving standard treatment.

"The study demonstrates the importance of working with patients and families to provide pragmatic, patient-centred approaches to care that are tailored to the affected individual's needs," says Freedman.

Hugh Thomas

ORIGINAL ARTICLE Freedman, S. B et al. Effect of dilute apple juice and preferred fluids versus electrolyte maintenance solution on treatment failure among children with mild gastroenteritis: a randomized clinical trial.JAMA 315, 1966-1974 (2016) 